\title{
Metacognitive and social cognition training (MSCT) in schizophrenia: A preliminary efficacystudy
}

\author{
Nuno B.F. Rocha, Cristina Queirós
}

\begin{abstract}
Psychosocial interventions have proven to be effective in treating social cognition in people with psychotic disorders. The current study aimed to determine the effects of a metacognitive and social cognition training (MSCT) program, designed to both remediate deficits and correct biases in social cognition. Thirty-five clinically stable outpatients were recruited and assigned to the MSCT program ( $\mathrm{n}=19$ ) for 10 weeks (18 sessions) or to the TAU group ( $\mathrm{n}=16$ ), and they all completed pre- and post-treatment assessments of social cognition, cogni- tive biases, functioning and symptoms. The MSCT group demonstrated a significant improvement in theory of mind, social perception, emotion recognition and social functioning. Additionally, the tendency to jump to conclusions was significantly reduced among the MSCT group after training. There were no differential benefits regarding clinical symptoms except for one trend group effect for general psychopathology. The results support the efficacy of the MSCT format, but further development of the training program is required to increase the benefits related to attributional style.
\end{abstract}

Keyw ords: Schizophrenia Social cognition Rehabilitation Cognitive bias Metacognition

\section{Introduction}

Impairments in social cognition are a consistent finding among patients with schizophrenia. These can be conceptualized both as deficits and as biases. Most deficits are found in theory of mind, emotion recognition and social perception (Green et al., 2005, 2008), while biases include a broad array of cognitive distortions such as attributional and jumping to conclusion biases that lead to failures in data acquisition and attribution of causes. These biases appear to be related to the formation and maintenance of symptoms that are characteristic of schizophrenia, particularly delusions (Garety and Freeman, 1999; Freeman, 2007; Moritz and Woodward, 2007).

Social cognitive processes are unique determinants of functional outcomes in schizophrenia (Couture et al., 2006). These processes can assume one of two roles. First, there is growing evidence that social cognition is a direct predictor of functional outcomes and that it determines community functioning to a greater extent than does neurocognition (Fett et al., 2011). Second, social cognition has been found to mediate the relationship between basic neurocognition and functional outcome, thereby making it more proximal to daily life functioning (Vauth et al., 2004; Sergi et al., 2007; Couture et al., 2011). Accordingly, social cognition should be considered an important target for intervention.
Several psychosocial interventions have been developed to overcome and correct the social cognitive features of schizophrenia. Most of these treatment programs were designed to directly address deficits in social cognition (e.g., emotion recognition, theory of mind) using remediation methods, while debiasing approaches are rarely employed when the primary focus is social cognitive processes (e.g., Hogarty et al., 2004; Roncone et al., 2004; Silver et al., 2004; Wolwer et al., 2005; Choi and Kwon, 2006; Kayser et al., 2006; Russell et al., 2006; Marsh et al., 2010; Mazza et al., 2010). However, deficits and biases affecting social cognition occur together in schizophrenia, and thus potentiate each other. For that reason, we hypothesize that the integration of approaches designed to target deficits and to provide corrective experiences may be most advantageous not only for biases but for the amelioration of symptoms of psychosis as well.

Thus, the aim of this study was to determine the effects of a metacognitive and social cognition training (MSCT) program designed to both remediate deficits and to correct biases in social cognition. The MSCT may be regarded as a hybrid of two well-known treatment formats: social cognition programs (e.g., social cognition and interaction training; social cognitive skills training) (Penn et al., 2007; Horan et al., 2009; Roberts and Penn, 2009) and metacognitive training for schizophrenia (Moritz et al., 2010b). While both treatment formats address similar skills, they apply different approaches. Social cognition programs usually make use of direct training of social cognitive processes such as emotion perception, attributional bias and theory of mind, while metacognitive training aims to raise the patient's awareness of the presence and dysfunctionality of cognitive distortions and providing corrective experiences. For example, while social cognition 

programs offer training strategies to help in the identification of basic emotions, metacognitive training focuses on the susceptibility to misinterpret and make false assumptions based on facial expressions when contextual information is scarce. Furthermore, the outcomes for both programs differ. While social cognition programs tend to emphasize improvement on social cognitive tests (e.g., Combs et al., 2007a), metacognitive training usually demonstrates the potential to reduce symptoms (particularly delusions) and biases (e.g., Moritz et al., 2011a).

To our knowledge, these different yet complementary approaches were never used in combination. We compared MSCT plus treatmentas-usual (TAU) to TAU among outpatients with schizophrenia in a quasi-experimental trial to evaluate whether this intervention package results in improvements in social cognition and functioning and in a reduction in symptoms.

\section{Materials and methods}

\subsection{Participants}

Participants were recruited from three socio-occupational centers (forums sócio-ocupacionais) that provide services to individuals with stable, severe and persistent mental illnesses. The centers were located in the districts of Porto (AFUA and ANARP) and Aveiro (Casa Ozanam), Portugal. Patients were included if they had a diagnosis of schizophrenia based on the DSM-IV criteria as determined by the patient's psychiatrist, were aged between 20 and 55 years, and were clinically stable without significant changes in medication for at least one month. The patients with diagnoses of neurologic disorders or with current substance dependence were excluded from the study.

This study was approved by the Scientific Committee of the Faculdade de Psicologia e de Ciências da Educação da Universidade do Porto and by the directive board of the AFUA, ANARP and Casa Ozanam. All participants gave written informed consent, and there was no financial compensation for participation.

Thirty-five clinically stable outpatients were recruited and assigned to either the MSCT program ( $\mathrm{n}=19$; three females) or to the treatment-as-usual (TAU; $\mathrm{n}=16$; one female) group. Participants were assigned to the TAU group if they met study criteria, declined to participate in the MSCT program or were unable to attend to the sessions. Participants from the MSCT group attended at least $80 \%$ of the program sessions. No differences were found between groups regarding socio-demographic or clinical characteristics (Table 1).

\section{Table 1}

Summary of participant characteristics and baseline performance.

\begin{tabular}{|c|c|c|c|}
\hline Characteristics & $\begin{array}{l}\text { MSCT }(\mathrm{n}=19) \\
\text { M(S.D.) }\end{array}$ & $\begin{array}{l}\text { TAU (n = 16) } \\
\text { M(S.D.) }\end{array}$ & $\mathrm{p}$ \\
\hline Fonder (F/M) & $3 / 16$ & $1 / 15$ & $61^{\mathrm{a}}$ \\
\hline Age (years) & $38.63(8.88)$ & $35.94(8.69)$ & $.37^{\mathrm{b}}$ \\
\hline \multicolumn{4}{|l|}{ Educationallevel } \\
\hline b9years & 6 & 9 & $.21^{\mathrm{c}}$ \\
\hline $9-12$ years & 10 & 7 & \\
\hline Higher education & 3 & 0 & \\
\hline \multicolumn{4}{|l|}{ Maritalstatus } \\
\hline Married & 2 & 0 & $.62^{\mathrm{c}}$ \\
\hline Single & 14 & 15 & \\
\hline Divorced/separated & 2 & 1 & \\
\hline Deceased spouse & 1 & 0 & \\
\hline Length of illness (years) & $13.68(7.49)$ & $13.63(8.97)$ & $.98^{\mathrm{b}}$ \\
\hline Chlorpromazine equivalent dose ( $\mathrm{mg} /$ day) & $406.98(225.45)$ & $432.79(322.89)$ & $.78^{\mathrm{b}}$ \\
\hline
\end{tabular}

\subsection{Instruments}

Participants of both groups (MSCT and TAU) were evaluated twice, once before and once after training (up to two weeks after training). The test battery consisted of social cognitive and cognitive biases as well as clinical and functional scales.

\subsubsection{Social cognition and cognitive biases}

Emotion recognition was measured using the Facial Emotion Identification Test (FEIT; Kerr and Neale, 1993), which includes 19 pictured faces displaying basic emotions that the participant must correctly identify. Total scores range from 0 to 19 .

Emotion regulation was measured using the managing emotions section of the Mayer-Salovey-Caruso Emotional Intelligence Test (MSCEIT-ME; Mayer et al., 2002), which has been recommended by the NIMH initiative titled Measurement and Treatment Research to Improve Cognition in Schizophrenia (Nuechterlein and Green, 2006). Participants are required to judge the efficacy of different responses for achieving goals or for maintaining or regulating emotions in different situations. A total score was derived using the MSCEIT general consensus method.

Theory of mind was measured using the hinting task (Corcoran et al., 1995), which consists of ten written vignettes, each of which presents an interaction between two characters and ends with one of the characters uttering a hint. The participant's task is to determine what the character really meant when he/she said this. Total scores range from 0 to 20 .

Attributional style was assessed using the Ambiguous Intentions Hostility Questionnaire - ambiguous situations (AIHQ-A; Combs et al., 2007b). Participants read five short second-person vignettes describing ambiguous social situations and then answer questions about the intentions of the characters and how the subjects themselves would respond to the situation. The AIHQ provides hostility and aggression bias scores, along with a composite blame score (average of intentionality, anger, and blame item ratings).

Social perception was measured using the Social Perception Scale (SPS; García et al., 2003). This measure consists of four photographs that are presented one at a time to the participant. The participants are then asked to carefully look at the picture for $2 \mathrm{~min}$. Following the methods of other studies using the SPS (e.g., Combs et al., 2007a), performance is indexed as the total number of accurate details identified in the picture.

Jumping to conclusion (JTC) bias was measured using the fish task (Moritz et al., 2010a; Speechley et al., 2010). Participants were shown two lakes with two different colors of fish in opposite ratios $(80 \%$ and $20 \%$ ). Participants were told that fish would be caught one-by-one from only one of the two lakes. The participants were then instructed to decide from which of the two lakes the fish were caught. Draws to decision (DTD) correspond to the number of fish a participant required before deciding and JTC was operationalized in this study as making a decision after the first fish. For the post-assessment, a second version with different colors but the same ratios was used.

The Trail Making Test B (TMT-B; Reitan, 1992; Reitan and Wolfson, 1995) was also included to attend to changes in cognitive flexibility during the MSCT program. In this task, participants must switch between the numerical mode and the alphabetic mode by connecting 26 numbers and characters. The score is the total time needed to finish the task. To keep the assessment schedule brief, we did not include any additional neurocognitive testing.

\subsubsection{Functional outcome and clinical}

Functioning was determined using the life skills profile (LSP; Rosen et al., 1989). The LSP includes 39 items divided into five subscales: self-care, non-turbulence, social contact, communication and responsibility. The severity of schizophrenia-related symptoms was assessed using the Positive and Negative Syndrome Scale (PANSS; Kay et al., 1987). 


\subsection{Intervention}

The participants in the experimental condition underwent a 10week metacognitive and social cognition training (MSCT) designed to address social-cognitive biases (e.g., jumping to conclusions, selfserving bias, bias against disconfirmatory evidence, need for closure and liberal acceptance) and other aspects of social cognition (emotion recognition, theory of mind and social perception). The MSCT is comprised of 18 sessions: one session each in weeks one and ten, and two sessions each in weeks two through nine. Session 1 addresses the relationship among emotions, thoughts and behaviors, focusing on mistakes that can be made in the inference of causality in social situations. During weeks two through nine, the first session of each week is a metacognitive psychoeducational session, and the second is an interactive social cognition remediation session. In the final session of the MSCT, participants report the application of the program to particular situations in their own daily lives.

Each metacognitive psychoeducational session is based on the Portuguese version of the metacognitive program developed by Moritz and colleagues (Moritz et al., 2010b,c), which can be obtained online at no cost at www.uke.de/mct. All sessions include exercises to train participants in specific ways to process information and to illustrate cognitive biases as well as a set of case examples and slides underlining the relevance of particular biases to daily life. Concluding remarks are always provided to show how exaggerations of (normal) thinking biases can lead to problems in daily life and may sometimes culminate in harmful suspicions and delusions.

The interactive social cognition remediation sessions focus on the processing of emotional and social cues, the analysis of social situations, the understanding of non-literal speech or deception and the ability to take another's perspective. For these purposes, we incorporated several exercises and didactic materials designed to teach social cognitive strategies. Materials and exercises were either originally developed by our team or were inspired by other wellknown training programs (e.g., Hogarty et al., 2004; Roberts and Penn, 2009). The feasibility of these materials and exercises were previously tested in a clinical intervention with other patients in a non-research set-up and/or with undergraduate students of the Porto Polytechnic Institute. These materials and exercises include training exercises to direct the attention to relevant facial features and mimicry to potentiate the ability to recognize emotions; presentations with film clips and photos to train in the appreciation of social context and the analysis of social cognitive stimuli by separating facts from assumptions; exercises to determine whether others are using sarcasm, humor or deception, particularly in ambiguous situations, and; group dynamics fostering the ability to take another's perspective (e.g., condensed messages, back-to-back drawings). Each session is complemented with guided discussion and homework.

The TAU consisted of regular medication and a set of psychosocial services provided at each socio-occupational center. These services included life and social skills training, psychoeducational groups, leisure activity suggestions, stress management, family education and support, and individual psychotherapy. As the TAU differed across participants according to each participant's own rehabilitation plan, it was not influenced by the study procedures.

\subsection{Statistical analysis}

Group differences were tested by comparing post-test scores between the two groups using one-way analyses of covariance (ANCOVA). Post-treatment scores were used as the dependent variables with the group entered as a fixed variable. Pre-test scores served as covariates to adjust for baseline. Effect sizes were calculated for within (Cohen's d) and between group (partial eta squared $-\eta_{\text {partia }}^{2}$ ) changes.

\section{Results}

The MSCT patients and the TAU patients did not significantly differ in social cognition, symptoms or functioning at baseline, except for responsibility on the life skills profile $(t=-2.12 ; p=.04)$.

Descriptive data, results of ANCOVA and between-group and within-group effect sizes are presented in Table 2. Regarding functioning, ANCOVA revealed significant differences in social contact, communication and total score on the LSP, with small within-group effect sizes (d) in favor of the MSCT group. These effects of the MSCT on functioning were mediated by a worsening in the TAU group. Regarding symptoms, there was only one trend group effect for PANSS general psychopathology with medium between-group $\left(\eta_{\mathrm{p}}{ }^{2}\right)$ and small within-group effect sizes.

Among social cognitive measures, ANCOVA revealed significant differences for theory of mind, social perception and emotion recognition that favor the MSCT, with large between- and within-group effect sizes. The tendency to jump to conclusions according to the DTD index was significantly reduced after training in the MSCT group, yielding medium between-group and large within-group effect sizes.

No differences were detected between groups on emotion regulation, attributional style or cognitive flexibility. Within-group effect sizes on the MSCT were small for emotion regulation, hostility bias and aggression bias and medium for the blame bias.

\section{Discussion}

This study provides preliminary evidence for the efficacy of combining remediation and debiasing approaches to target social cognition in individuals with schizophrenia. Following intervention, the MSCT group showed improvements in several social cognition measures. The most notable improvements were in theory of mind, emotion recognition and social perception. There was also an increase in the number of draws before making a decision on the fish task, which is an indicator of a decrease in jumping to conclusions. The MSCT group also showed a small but significant increase in psychosocial functioning, especially regarding social contact and communication, which are areas of functioning closely related to social cognition skills. Finally, participation in the MSCT program plus TAU was accompanied by reductions in general schizophreniarelated symptoms.

In a recent meta-analysis, Kurtz and Richardson (2012) found significant effects for social cognition intervention on emotion recognition and theory of mind, which is consistent with the results obtained and reported herein. The largest magnitude of effect was found for emotion recognition in both studies.

Several studies have shown that it is possible to recover the ability to recognize emotions with well-defined strategies and structured training (Frommann et al., 2003; Silver et al., 2004; Wolwer et al., 2005; Russell et al., 2006; Combs et al., 2007a; Russell et al., 2008; Roberts and Penn, 2009; Marsh et al., 2010; Mazza et al., 2010; Roberts et al., 2010; Combs et al., 2011). In comprehensive training programs that address emotion perception as well as other dimensions, such as theory of mind and attributions, emotion recognition improvements are typically observed even when no changes are observed in other social cognition areas (Horan et al., 2009). Emotion perception is the first skill addressed in the interactive social cognition remediation sessions of the MSCT, and when questioned, most participants referred to this area as one of the most important themes of the intervention program.

Regarding the results obtained with respect to theory of mind, the results of the MSCT confirm that although theory of mind involves complex processes to estimate the mental states of others, which is notoriously difficult to translate into training exercises (Horan et al., 2009), it is, in fact, possible to improve meta-representation and perspective in patients with schizophrenia. 
Comparisons between the scores of pre- and post-experimental sessions for each group. The results are given as the mean (standard deviation)

\begin{tabular}{|c|c|c|c|c|c|c|c|c|c|c|}
\hline & \multicolumn{2}{|l|}{$\operatorname{MSCT}(\mathrm{n}=21)$} & \multirow{2}{*}{$\begin{array}{l}\text { Estimated } \\
\text { mean }\end{array}$} & \multicolumn{2}{|l|}{ TAU $(\mathrm{n}=16)$} & \multirow{2}{*}{$\begin{array}{l}\text { Estimated } \\
\text { mean }\end{array}$} & \multirow[t]{2}{*}{$\mathrm{F}$} & \multicolumn{3}{|c|}{ Effect sizes } \\
\hline & Pre-treatment & Post-treatment & & Pre-treatment & Post-treatment & & & $\begin{array}{l}\text { Between } \\
\text { group } \eta_{p}^{<}\end{array}$ & $\begin{array}{l}\text { Within MSCT } \\
\text { group d }\end{array}$ & $\begin{array}{l}\text { Within TAU } \\
\text { group d }\end{array}$ \\
\hline \multicolumn{11}{|c|}{ Social cognition and cognitive biases } \\
\hline Hinting task & $15.58(3.61)$ & $17.95(2.34)$ & 17.15 & $13.06(5.01)$ & $13.94(5.40)$ & 14.89 & $5.42^{*}$ & .15 & .80 & .17 \\
\hline Fish task DTD & $2.47(1.95)$ & $4.05(2.74)$ & 3.82 & $1.88(1.26)$ & $2.00(1.67)$ & 2.27 & $5.78^{*}$ & .15 & .68 & .08 \\
\hline JTC \% & $47.37 \%$ & $26.32 \%$ & & $56.25 \%$ & $68.75 \%$ & & & & & \\
\hline SPS & $21.37(8.37)$ & $28.21(7.61)$ & 27.85 & $19.06(8.96)$ & $21.06(9.01)$ & 21.49 & $5.60^{\star}$ & .15 & .88 & .23 \\
\hline MSCEIT-ME & $80.64(8.78)$ & $82.39(11.06)$ & 82.21 & $80.11(8.61)$ & $78.92(7.57)$ & 79.13 & 1.52 & .05 & .18 & -.15 \\
\hline FEIT & $11.84(3.20)$ & $14.53(2.12)$ & 14.32 & $10.69(3.67)$ & $11.56(3.35)$ & 11.81 & $8.99^{* *}$ & .22 & 1.02 & .26 \\
\hline AIHQ-A hostility & $9.58(2.41)$ & $8.89(2.31)$ & 8.89 & $9.38(2.87)$ & $9.13(1.96)$ & 9.13 & .11 & .00 & .30 & .11 \\
\hline Blame & $15.51(4.20)$ & $13.68(3.27)$ & 13.82 & $16.63(3.70)$ & $15.02(3.14)$ & 14.86 & .97 & .03 & .50 & .49 \\
\hline Aggression & $9.56(2.25)$ & $9.68(2.69)$ & 9.79 & $10.06(1.53)$ & $10.56(2.25)$ & 10.45 & .62 & .02 & -.05 & -.27 \\
\hline TMT-B & $119.32(72.87)$ & $106.46(67.21)$ & 115.76 & $158.02(113.95)$ & $142.88(86.58)$ & 131.85 & .61 & .02 & .19 & .15 \\
\hline \multicolumn{11}{|c|}{ Functional outcome and clinical } \\
\hline LSP self-care & $31.26(4.20)$ & $31.58(4.66)$ & 30.75 & $29.50(6.83)$ & $29.31(7.16)$ & 30.30 & .70 & .02 & .07 & -.03 \\
\hline Non-turbulence & $42.68(5.38)$ & $42.74(5.49)$ & 43.21 & $43.69(4.78)$ & 43.75 (5.18) & 43.19 & .01 & .00 & .01 & .01 \\
\hline Social contact & $12.89(3.11)$ & $13.37(2.95)$ & 14.19 & $14.63(3.77)$ & $13.88(4.60)$ & 12.91 & $6.95^{*}$ & .18 & .16 & -.18 \\
\hline Communication & $19.89(3.13)$ & $20.47(3.08)$ & 20.47 & $19.88(3.10)$ & 19.13(2.99) & 19.13 & $7.50^{*}$ & .19 & .19 & -.25 \\
\hline Responsibility & $18.84(1.68)$ & $19.00(1.29)$ & 18.38 & $17.38(2.31)$ & $17.44(2.45)$ & 18.17 & 1.19 & .04 & .11 & .03 \\
\hline Total score & $125.58(11.32)$ & $127.16(12.82)$ & 126.91 & $125.06(16.45)$ & $123.50(17.36)$ & 123.80 & $6.41^{*}$ & .17 & .18 & -.10 \\
\hline PANSS negative & $21.11(6.49)$ & $20.74(6.65)$ & 20.75 & $21.13(5.03)$ & $20.94(5.14)$ & 20.93 & .04 & .00 & .06 & -.04 \\
\hline Positive & $17.84(6.69)$ & $16.84(7.04)$ & 15.38 & $14.50(4.91)$ & $13.44(4.68)$ & 15.18 & .07 & .00 & .15 & .23 \\
\hline General & $44.0(10.90)$ & 41.63 (9.69) & 39.36 & $38.06(10.11)$ & $39.13(9.21)$ & 41.83 & $4.1^{* \star \star}$ & .11 & .24 & -.11 \\
\hline Total & $82.95(19.20)$ & $79.21(18.65)$ & 75.46 & 73.69 (18.98) & $73.50(16.79)$ & 77.96 & 1.58 & .05 & .20 & .01 \\
\hline
\end{tabular}

$* \mathrm{p} b .05$.

** p b.01.

$\star * \star \mathrm{p} b .06$.

Unlike Kurtz and Richardson (2012), we found significant changes in social perception. These results reflect the MSCT's focus on the collection of relevant contextual information before formulating interpretations and conclusions. This distinctive feature, which is emphasized across the MSCT sessions, seems to have produced a positive effect that warrants replication in future research.

With respect to attributions, improvement may require different techniques or greater treatment dosage. As in the meta-analysis of Kurtz and Richardson (2012), we found no significant changes in the biases of hostility, blame and aggression. Most attributional changes resulting from training programs on social cognition occurred in inpatients. Thus, the changes may have occurred as a result of the effects of clinical improvements (Combs et al., 2007a; Penn et al., 2007).

The MSCT training exerted a beneficial effect on JTC, which is a positive result considering that JTC in schizophrenia has been resistant to change after other psychological interventions, such as cognitive-behavioral therapy (Garety et al., 2008). JTC bias is thought to play a role in the formation and maintenance of delusions in patients with schizophrenia based on a propensity to hold implausible beliefs with unwarranted conviction (McKay et al., 2006). Thus, it is possible that the MSCT may have a beneficial anti-psychotic effect that complements psychopharmacology, such as metacognitive training (Moritz et al., 2011b).

We did not find any benefits of the MSCT regarding cognitive flexibility, unlike the findings of Combs et al. (2007a) with inpatients. This may be because our participants were outpatients and because the primary targets of the program are deficits in social cognition and cognitive biases rather than neurocognition. Another possible reason is that, to keep the assessment schedule brief, we did not include more neurocognitive tests other than the Trail Making Test B. This may have prevented the capturing of changes in neurocognition during the MSCT program.

In addition to the findings regarding the social cognitive variables, the MSCT improved functioning, particularly with respect to the social and communication domains. Although effect sizes were small, this finding is quite encouraging given that the main goal of the MSCT is to improve social functioning in patients with schizophrenia. These results are consistent with findings that social cognition is associated with functional outcomes of schizophrenia and support the hypothesis that the amelioration of social cognition can produce positive functional outcomes (Fett et al., 2011). However, these results should be interpreted cautiously as the effects of the MSCT on functioning were accompanied by a worsening in the TAU group.

Contrary to expectation, we observed no effect of the MSCT on positive symptoms. One possibility is that the treatment period was not long enough to detect sustained improvements. Alternatively, effects may have been observed if our psychopathological assessment used a more sensitive symptom scale, such as the Psychotic Symptom Rating Scales (Haddock et al., 1999). Still, these findings are similar to those in the meta-analysis conducted by Kurtz and Richardson (2012), which reported an absence of effects on positive and negative symptoms as a result of social cognition rehabilitation. On the other hand, we detected a trend toward a positive significant effect of the MSCT in decreasing general psychopathology, which was the main positive clinical outcome reported by Kurtz and Richardson (2012).

We must infer that there is a possibility that the outcomes of any social cognitive training (or even cognitive remediation) may result, to some extent, in a "train to the test" effect. Particularly, emotional recognition training materials are frequently similar to those used in the assessment, which may cause higher effect sizes. As the MSCT is not an exception to this fact, some of the results may suffer from this effect, a factor that should be carefully examined in future research. Nevertheless, the effects of the MSCT on functioning may be evidence of the potential of this training format to produce generalizable benefits.

Limitations of this preliminary report include the small sample size as it limited the power to detect significant differences. Additional limitations included the use of a quasi-experimental design and the lack of a placebo condition, which limited control for non-specific elements of the training program. Finally, the absence of a follow-up period prevented analysis of whether the training effects are durable and of the long-term benefits regarding clinical and functional outcomes.

In closing, the social cognitive improvements associated with the MSCT encourage the application of a treatment format that integrates 
remediation and debiasing approaches to address social cognition in patients with schizophrenia. The findings support continued research into the efficacy of the MSCT, and controlled research is needed to establish the MSCT program format.

\section{Role of funding source}

This study was supported by grant SFRH/BD/42290/2007 from the Fundação para a Ciência e a Tecnologia (Portugal) and by a PRODOC grant from the Instituto Politécnico do Porto.

These funding sources had no further role in the study design; in the collection, analysis and interpretation of data; in the writing of the report; or in the decision to submit this manuscript for publication.

\section{Contributors}

NR and CQ designed the study, collected the data and conducted the statistical analysis; undertook the data analysis and interpretation; contributed to and have approved the final manuscript.

\section{Conflict of interest}

The authors do not have any conflicts of interest.

\section{Acknowledgment}

We thank David Roberts (University of Texas Health Science Center) and Steffen Moritz (University Medical Center Hamburg-Eppendorf) for valuable consultations.

\section{References}

Choi, K., Kwon, J., 2006. Social cognition enhancement training for schizophrenia: a preliminary randomized controlled trial. Community Ment. Health J. 42 (2), 177-187.

Combs, D., Adams, S., Penn, D., Roberts, D., Tiegreen, J., Stem, P., 2007a. Social cognition and interaction training (SCIT) for inpatients with schizophrenia spectrum disorders: preliminary findings. Schizophr. Res. 91 (1-3), 112-116.

Combs, D., Penn, D., Wicher, M., Waldheter, E., 2007b. The Ambiguous Intentions Hostility Questionnaire (AIHQ): a new measure for evaluating hostile social-cognitive biases in paranoia. Cogn. Neuropsychiatry 12 (2), 128-143.

Combs, D., Chapman, D., Waguspack, J., Basso, M., Penn, D., 2011. Attention shaping as a means to improve emotion perception deficits in outpatients with schizophrenia and impaired controls. Schizophr. Res. 127 (1-3), 151-156.

Corcoran, R., Mercer, G., Frith, C., 1995. Schizophrenia, symptomatology and social inference: investigating "theory of mind" in people with schizophrenia. Schizophr. Res. 17, 5-13.

Couture, S., Penn, D., Roberts, D., 2006. The functional significance of social cognition in schizophrenia: a review. Schizophr. Bull. 32 (s1), S44-S63.

Couture, S., Granholm, E., Fish, S., 2011. A path model investigation of neurocognition, theory of mind, social competence, negative symptoms and real-world functioning in schizophrenia. Schizophr. Res. 125 (2-3), 152-160.

Fett, A., Viechtbauer, W., Dominguez, M., Penn, D., van Os, J., Krabbendam, L., 2011. The relationship between neurocognition and social cognition with functional outcomes in schizophrenia: a meta-analysis. Neurosci. Biobehav. Rev. 35 (3), 573-588.

Freeman, D., 2007. Suspicious minds: the psychology of persecutory delusions. Clin. Psychol. Rev. 27, 425-457.

Frommann, N., Streit, M., Wolwer, W., 2003. Remediation of facial affect recognition impairments in patients with schizophrenia: a new training program. Psychiatry Res. 117 (3), 281-284.

García, S., Fuentes, I., Ruíz, J.C., Gallach, E., Roder, V., 2003. Application of the IPT in a Spanish sample: evaluation of the "Social Perception Subprogramme". Int. J. Psychol. Psychol. Ther. 3 (2), 299-310.

Garety, P., Freeman, D., 1999. Cognitive approaches to delusions: a critical review of theories and evidence. Br. J. Clin. Psychol. 38, 113-154.

Garety, P., Fowler, D., Freeman, D., Bebbington, P., Dunn, G., Kuipers, E., 2008. Cognitivebehavioural therapy and family intervention for relapse prevention and sym-ptom re- duction in psychosis: randomised controlled trial. Br. J. Psychiatry 192 (6), 412-423. Green, M.

Olivier, B., Crawley, J., Penn, D., Silverstein, S., 2005. Social cognition in schizophrenia: recommendations from the measurement and treatment research to improve cognition in schizophrenia new approaches conference. Schizophr. Bull. 31 (4), 882-887.

Green, M., Penn, D., Bentall, R., Carpenter, W., Gaebel, W., Gur, R., Kring, A., Park, S., Silverstein, S., Heinssen, R., 2008. Social cognition in schizophrenia: an NIMH workshop on definitions, assessment, and research opportunities. Schizophr. Bull. 34 (6), 1211-1220

Haddock, G., McCarron, J., Tarrier, N., Faragher, E., 1999. Scales to measure dimensions of hallucinations and delusions: the psychotic symptom rating scales (PSYRATS). Psychol. Med. 29 (4), 879-889.

Hogarty, G., Flesher, S., Ulrich, R., Carter, M., Greenwald, D., Pogue-Geile, M., Kechavan, M., Cooley, S., DiBarry, A., Garrett, A., Parepally, H., Zoretich, R., 2004. Cognitive enhancement therapy for schizophrenia: effects of a 2-year randomized trial on cognition and behavior. Arch. Gen. Psychiatry 61 (9), 866-876.
Horan, W., Kern, R., Shokat-Fadai, K., Sergi, M., Wynn, J., Green, M., 2009. Social cognitive skills training in schizophrenia: an initial efficacy study of stabilized outpatients. Schizophr. Res. 107 (1), 47-54.

Kay,S., Fiszbein,A., Opler, L., 1987. The Positiveand NegativeSyndrome Scale(PANSS) for schizophrenia. Schizophr. Bull. 13 (2), 261-276.

Kayser, N., Sarfati, Y., Besche, C., Hardy-Baylé, M., 2006. Elaboration of a rehabilitation method based on a pathogenetic hypothesis of "theory of mind" impairment in schizophrenia. Neuropsychol. Rehabil. 16 (1), 83-95.

Kerr, S., Neale, J., 1993. Emotion perception in schizophrenia: specific deficit or further evidence of generalized poor performance? J. Abnorm. Psychol. 102 (2), 312-318.

Kurtz, M., Richardson, C., 2012. Social cognitive training for schizophrenia: a metaanalytic investigation of controlled research. Schizophr. Bull. 38 (5), 1092-1104.

Marsh, P., Green, M., Russell, T.A., McGuire, J., Harris, A., Coltheart, M., 2010. Remediation of facial emotion recognition in schizophrenia: functional predictors, generalizability, and durability. Am. J. Psychiatr. Rehabil. 13 (2), 143-170.

Mayer, J., Salovey, P., Caruso, D., 2002. Mayer-Salovey-Caruso Emotional Intelligence Test (MSCEIT) User's Manual. Multi-Health Systems, Toronto.

Mazza, M., Lucci, G., Pacitti, F., Pino, M., Mariano, M., Casacchia, M., Roncone, R., 2010. Could schizophrenic subjects improve their social cognition abilities only with observation and imitation of social situations? Neuropsychol. Rehabil. 20 (5), 675703.

McKay, R., Langdon, R., Coltheart, M., 2006. Need for closure, jumping to conclusions, and decisiveness in delusion-prone individuals. J. Nerv. Ment. Disord. 194 (6), 422-426.

Moritz, S., Woodward, T., 2007. Metacognitive training in schizophrenia: from basic research to knowledge translation and intervention. Curr. Opin. Psychiatry 20, 619-625.

Moritz, S., Veckenstedt, R., Hottenrott, B., Woodward, T., Randjbar, S., Lincoln, T., 2010a. Different sides of the same coin? Intercorrelations of cognitive biases in schizophrenia. Cogn. Neuropsychiatry 15 (4), 406-421.

Moritz, S., Vitzthum, F., Randjbar, S., Veckenstedt, R., Woodward, T., 2010b. Detecting and defusing cognitive traps: metacognitive intervention in schizophrenia. Curr. Opin. Psychiatry 23, 561-569.

Moritz, S., Woodward, T., Rocha, N., 2010c. Treino Metacognitivo para pacientes com Esquizofrenia (TMC) - Manual. VanHam Campus Press, Hamburg.

Moritz, S., Kerstan, A., Veckenstedt, R., Randjbar, S., Vitzthum, F., Schmidt, C., Heise, M., Woodward, T., 2011a. Further evidence for the efficacy of a metacognitive group training in schizophrenia. Behav. Res. Ther. 49 (3), 151-157.

Moritz, S., Veckenstedt, R., Randjbar, S., Vitzthum, F., Woodward, T., 2011b. Antipsychotic treatment beyond antipsychotics: metacognitive intervention for schizophrenia patients improves delusional symptoms. Psychol. Med. 41 (9), 1823-1832.

Nuechterlein, K., Green, M., 2006. MCCB: MATRICS Consensus Cognitive Battery. MATRICS Assessment Inc., Los Angeles.

Penn, D., Roberts, D., Combs, D., Sterne, A., 2007. Best practices: the development of the social cognition and interaction training program for schizophrenia spectrum disor ders. Psychiatr. Serv. 58 (4), 449-451.

Reitan, R., 1992. Trail Making Test: Manual for Administration and Scoring. Reitan Neuropsychological Laboratories, Tuscon, AZ.

Reitan, R., Wolfson, D., 1995. Category test and trail making test as measures of frontal lobe functions. Clin. Neuropsychol. 9, 50-56.

Roberts, D., Penn, D., 2009. Social cognition and interaction training (SCIT) for outpatients with schizophrenia: a preliminary study. Psychiatry Res. 166 (2-3), 141-147.

Roberts, D., Penn, D., Labate, D., Margolis, S., Sterne, A., 2010. Transportability and feasibility of social cognition and interaction training (SCIT) in community settings. Behav. Cogn. Psychother. 38 (1), 35-47.

Roncone, R., Mazza, M., Frangou, I., Risio, A.D., Ussorio, D., Tozzini, C., Casacchia, M., 2004. Rehabilitation of theory of mind deficit in schizophrenia: a pilot study of metacognitive strategies in group treatment. Neuropsychol. Rehabil. 14 (4), 421435

Rosen, A., Hadzi-Pavlovic, D., Parker, G., 1989. The life skills profile: a measure assessing function and disability in schizophrenia. Schizophr. Bull. 15 (2), 325-337.

Russell, T., Chu, E., Phillips, M., 2006. A pilot study to investigate the effectiveness of emotion recognition remediation in schizophrenia using the micro-expression training tool. Br. J. Clin. Psychol. 45 (Pt4), 579-583.

Russell, T., Green, M., Simpson, I., Coltheart, M., 2008. Remediation of facial emotion perception in schizophrenia: concomitant changes in visual attention. Schizophr. Res. 103 (1-3), 248-256.

Sergi, M., Rassovsky, Y., Widmark, C., Reist, C., Erhart, S., Braff, D., Marder, S., Green, M.F., 2007. Social cognition in schizophrenia: Relationships with neurocognition and negative symptoms. Schizophr. Res. 90, 316-324.

Silver, H., Goodman, C., Knoll, G., Isakov, V., 2004. Brief emotion training improves recognition of facial emotions in chronic schizophrenia. A pilot study. Psychiatry Res. 128, $147-154$.

Speechley, W., Whitman, J., Woodward, T., 2010. The contribution of hypersalience to the "jumping to conclusions" bias associated with delusions in schizophrenia. J. Psychiatry Neurosci. 35 (1), 7-17.

Vauth, R., Rüsch, N., Wirtz, M., Corrigan, P., 2004. Does social cognition influence the relation between neurocognitive deficits and vocational functioning in schizophrenia? Psychiatry Res. 128 (2), 155-165.

Wolwer, W., Frommann, N., Halfmann, S., Piaszek, A., Streit, M., Gaebel, W., 2005 Remediation of impairments in facial affect recognition in schizophrenia: efficacy and specificity of a new training program. Schizophr. Res. 80 (2-3), 295-303. 